\title{
INTEGRAL and Swift/XRT observations of the SFXT IGR J16479-4514: from quiescence to fast flaring activity (Research Note)
}

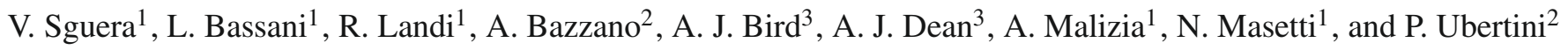 \\ ${ }^{1}$ IASF/INAF, via Piero Gobetti 101, 40129 Bologna, Italy \\ e-mail: sguera@iasfbo.inaf.it \\ 2 IASF/INAF, via Fosso del Cavaliere 100, 00133 Roma, Italy \\ 3 School of Physics and Astronomy, University of Southampton, Highfield SO17 1BJ, UK
}

Received 5 December 2007 / Accepted 26 April 2008

\section{ABSTRACT}

\begin{abstract}
Context. The fast X-ray transient IGR J16479-4514 is known to display flares typically lasting a few hours. Recently, its counterpart has been identified with a supergiant star, therefore the source can be classified as a member of the newly discovered class of Supergiant Fast X-ray Transients (SFXTs); specifically, it is the one with the highest duty cycle.

Aims. Characterize the quiescent X-ray behaviour of the source and compare its broad band spectrum to those during fast X-ray flares. Methods. We analysed IBIS and JEM-X data with OSA 5.1, along with archival Swift/XRT data.

Results. We present results from a long-term monitoring of IGR J16479-4514 with detailed spectral and timing information on 19 bright fast X-ray flares, 10 of which are newly discovered. We also report the first results on the quiescent X-ray emission. The typical luminosity value $\left(\sim 10^{34} \mathrm{erg} \mathrm{s}^{-1}\right)$ is about 2 orders of magnitude greater than what is typical of SFXTs, while its broad band $\mathrm{X}$-ray spectrum has a shape very similar to the shape during fast X-ray transient activity, i.e. a rather steep power law with $\Gamma \sim 2.6$. Conclusions. IGR J16479-4514 is characterised by a quiescent X-ray luminosity higher than what is typical of other known SFXTs but lower than persistent emission from classical SGXBs. We suggest that this source is a kind of transition object between these two systems, supporting the idea that there is a continuum of behaviour between the class of SFXTs and that of classical persistent SGXBs.
\end{abstract}

Key words. gamma rays: observations $-\mathrm{X}$-rays: binaries $-\mathrm{X}$-rays: bursts $-\mathrm{X}$-rays: individuals: IGR J16479-4514 X-rays: general

\section{Introduction}

Since its launch in 2002, the INTEGRAL satellite (Winkler et al. 2003) has played a key role in discovering many new high mass $\mathrm{X}$-ray binaries (HMXBs) thanks to its large field of view (FOV), continous monitoring of the galactic plane and good sensitivity. The majority of these systems turned out to be persistent supergiant high mass X-ray binaries (SGXBs) that escaped previous detection because of their very obscured nature (e.g. Walter et al. 2006). The remaining ones, named supergiant fast X-ray transients (SFXTs, Negueruela et al. 2006; Sguera et al. 2005, 2006), were missed before because of the very low level of quiescent X-ray luminosities $\left(\sim 10^{32}-10^{33} \mathrm{erg} \mathrm{s}^{-1}\right)$, occasionally interrupted by fast $\mathrm{X}$-ray flares lasting typically less than a day and reaching peak luminosities of $\sim 10^{36} \mathrm{erg} \mathrm{s}^{-1}$. This peculiar transient behaviour has never been seen before from classical persistent SGXBs, which are characterised by X-ray luminosities in the range $10^{36}-10^{38} \mathrm{erg} \mathrm{s}^{-1}$ with a few of them rarely displaying flaring activity on a timescale of a few hours, i.e. Vela X-1 (Staubert et al. 2004; Laurent et al. 1995). The fast X-rays flares from SFXTs should reflect inhomogeneities in the donor star stellar wind, which could be characterised by a clumpy nature (Negueruela et al. 2008a; Walter \& Zurita 2007; Leyder et al. 2007).

IGR J16479-4514 is one of the few SFXTs discovered so far by INTEGRAL. It was first detected during observations performed between August 8-10, 2003 (Molkov et al. 2003).
Subsequently, Sguera et al. $(2005,2006)$ unveiled its fast X-ray transient nature, reporting several fast flares strongly resembling those of confirmed SFXTs. Recently, Chaty et al. (2008) and Rahoui et al. (2008) have reported on optical and near/mid infrared observations of the source that led to identifying its counterpart with a supergiant star $(\mathrm{O} 8.5 \mathrm{I})$ at a distance of $\sim 4.9 \mathrm{kpc}$, hence its classification as an SFXT; specifically, it is the one with the highest duty cycle so far observed (Sguera et al. 2005, 2006; Walter \& Zurita 2007).

Here we report on the characteristics of 10 newly discovered fast flares detected by IBIS, and we provide for the first time $20-60 \mathrm{keV}$ spectral information for the set of 19 flares detected so far. For one such flare, we also report and discuss the broad band X-ray spectrum obtained by combining simultaneous Swift/XRT, JEM-X, and ISGRI data. Moreover we present, for the first time, broad band spectral data on the likely quiescent $\mathrm{X}$-ray emission of IGR J16479-4514 which is very rare information on SFXTs on account of their very recent discovery as a class of sources.

\section{Data analysis}

For this study, we have used data collected with IBIS/ISGRI (Ubertini et al. 2003; Lebrun et al. 2003) and JEM-X (Lund et al. 2003), the gamma-ray imager and the X-ray monitor onboard the INTEGRAL satellite. In particular, the IBIS data set 
Table 1. Summary of all IBIS detections of fast hard X-ray flares from IGR J16479-4514.

\begin{tabular}{|c|c|c|c|c|c|c|c|}
\hline N. & $\begin{array}{l}\text { Date } \\
\text { (UTC) }\end{array}$ & $\begin{array}{l}\text { Duration } \\
\text { (h) }\end{array}$ & $\begin{array}{c}\text { Peak flux } \\
(20-60 \mathrm{keV}, \mathrm{mCrab})\end{array}$ & $\begin{array}{l}\text { Peak luminosity } \\
\left(10^{36} \mathrm{erg} \mathrm{s}^{-1}\right)\end{array}$ & $\begin{array}{l}K T_{\mathrm{BR}} \\
(\mathrm{keV})\end{array}$ & $\Gamma$ & Ref. \\
\hline 1 & 5 Mar. 2003, 14:00 & $\sim 0.5$ & $\sim 560$ & $\sim 19$ & $21_{-2.5}^{+3}(0.56,14)$ & $2.9 \pm 0.2(0.87,14)$ & 1 \\
\hline 2 & 28 Mar. 2003, 8:30 & $\sim 1.5$ & $\sim 40$ & $\sim 1.3$ & & & 1 \\
\hline 3 & 21 Apr. 2003, 9:00 & $\sim 0.5 \ddagger$ & $\sim 100$ & $\sim 3.4$ & $21_{-11}^{+6}(0.6,14)$ & $3_{-0.6}^{+0.5}(0.54,14)$ & 1 \\
\hline 4 & 10 Aug. 2003, $\sim 12: 00$ & $\sim 60$ & $\sim 70$ & $\sim 2.4$ & & & 4 \\
\hline 5 & 14 Aug. 2003, 1:00 & $\sim 2 \ddagger$ & $\sim 40$ & $\sim 1.3$ & & & 1 \\
\hline 6 & 11 Aug. 2004, 7:00 & $\sim 8$ & $\sim 80$ & $\sim 2.7$ & $30_{-8}^{+15}(0.74,14)$ & $2.5 \pm 0.4(0.72,14)$ & 3 \\
\hline 7 & 15 Aug. 2004, 17:00 & $\sim 2$ & $\sim 55$ & $\sim 1.8$ & $\sim 40(1.1,14)$ & $2.3 \pm 0.9(1.06,14)$ & 3 \\
\hline 8 & 7 Sep. $2004, \sim 2: 00$ & $\sim 2$ & $\sim 80$ & $\sim 2.7$ & $44_{-14}^{+32}(0.65,14)$ & $2.2 \pm 0.3(0.65,14)$ & 2 \\
\hline 9 & 16 Sep. $2004, \sim 17: 00$ & $\sim 2.5$ & $\sim 120$ & $\sim 4$ & & $2.6 \pm 0.2(1.06,14)$ & 2 \\
\hline 10 & 27 Feb. 2005, 14:30 & $\sim 3$ & $\sim 75$ & $\sim 2.5$ & $46_{-17}^{+40}(0.93,20)$ & $2.2 \pm 0.45(1.01,20)$ & 3 \\
\hline 11 & 27 Mar. $2005, \sim 19: 00$ & $\sim 1$ & $\sim 40$ & $\sim 1.3$ & & & 3 \\
\hline 12 & 3 Apr. 2005, $\sim 00: 00$ & $\sim 2$ & $\sim 45$ & $\sim 1.5$ & & & 3 \\
\hline 13 & 4 Apr. $2005, \sim 03: 00$ & $\sim 9 \ddagger$ & $\sim 45$ & $\sim 1.5$ & $29_{-8}^{+15}(1.02,14)$ & $2.6 \pm 0.4(1.01,14)$ & 2 \\
\hline 14 & 9 Apr. 2005, 12:00 & $\sim 50$ & $\sim 60$ & $\sim 2$ & & & 3 \\
\hline 15 & 12 Aug. 2005, 19:00 & $\sim 9 \ddagger$ & $\sim 55$ & $\sim 1.8$ & $26_{-8.5}^{+21}(0.6,8)$ & $2.5 \pm 0.5(0.6,8)$ & 3 \\
\hline 16 & 17 Aug. 2005, 21:30 & $\sim 8$ & $\sim 40$ & $\sim 1.3$ & & & 3 \\
\hline 17 & 26 Aug. 2005, 05:00 & $\sim 6$ & $\sim 40$ & $\sim 1.3$ & & & 3 \\
\hline 18 & 30 Aug. 2005, $\sim 04: 00$ & $\sim 0.5$ & $\sim 180$ & $\sim 6$ & $38_{-11}^{+20}(0.65,19)$ & $2.3 \pm 0.35(0.8,19)$ & 5 \\
\hline 19 & 3 Mar. 2006, $\sim 09: 30$ & $\sim 3 \ddagger$ & $\sim 60$ & $\sim 2$ & & & 3 \\
\hline
\end{tabular}

$\sharp=$ Lower limit on the duration, $\star=$ assuming a distance of $\sim 4.9 \mathrm{kpc}$ (Chaty et al. 2008).

(1) Sguera et al. (2005); (2) Sguera et al. (2006); (3) this paper; (4) Molkov et al. (2003); (5) Kennea et al. (2005).

Table 2. Summary of Swift/XRT observations of IGR J16479-4514.

\begin{tabular}{|c|c|c|c|c|c|c|}
\hline No. & $\begin{array}{c}\text { Exposure } \\
\text { ks }\end{array}$ & $\begin{array}{l}\text { OBS date } \\
\text { (UTC) }\end{array}$ & $\begin{array}{c}\text { Average flux } \\
\left(1-9 \mathrm{keV}, \mathrm{erg} \mathrm{cm}^{-2} \mathrm{~s}^{-1}\right)\end{array}$ & $\begin{array}{l}\text { Average luminosity } \\
\left(\mathrm{erg} \mathrm{s}^{-1}\right)\end{array}$ & $\Gamma$ & $\begin{array}{c}N_{\mathrm{H}} \\
10^{22}\left(\mathrm{~cm}^{-2}\right)\end{array}$ \\
\hline OBS1 (decay flare N. 18) & 0.5 & 30 Aug. 2005 & $\sim 1.1 \times 10^{-10}$ & $\sim 3.2 \times 10^{35}$ & $\Gamma=1.1 \pm 0.8(0.99,20)$ & $8.5_{-4.5}^{+6.5}$ \\
\hline OBS1 (quiescence) & 8 & 30 Aug. 2005 & $\sim 6 \times 10^{-12}$ & $\sim 1.7 \times 10^{34}$ & $\Gamma=0.75 \pm 0.6(0.45,19)$ & $4.5_{-1.3}^{+2.2}$ \\
\hline $\begin{array}{l}\text { OBS2 } \\
\text { OBS3 }\end{array}$ & $\begin{array}{l}6.4 \\
4.1\end{array}$ & $\begin{array}{l}10 \text { Sep. } 2005 \\
14 \text { Sep. } 2005\end{array}$ & $\begin{array}{l}\sim 2.6 \times 10^{-11} \\
\sim 2 \times 10^{-12}\end{array}$ & $\begin{array}{l}\sim 7.4 \times 10^{34} \\
\sim 5.7 \times 10^{33}\end{array}$ & $\begin{array}{c}\Gamma=1.35 \pm 0.4(0.9,54) \\
\Gamma \sim 0.6(0.6,17)\end{array}$ & $\begin{array}{l}9.5_{-1.9}^{+2.2} \\
\sim 5\end{array}$ \\
\hline OBS4 & 5.4 & 18 Oct. 2005 & $\sim 7.5 \times 10^{-12}$ & $\sim 2.1 \times 10^{34}$ & $\Gamma=1.8 \pm 0.9(0.55,17)$ & $8.4_{-4}^{+5}$ \\
\hline
\end{tabular}

$\star$ Assuming a distance of $\sim 4.9 \mathrm{kpc}$ (Chaty et al. 2008; Rahoui et al. 2008).

consists of $\sim 2250$ pointings or science windows (ScWs, $\sim 2000 \mathrm{~s}$ duration) where IGR J16479-4514 was within $12^{\circ}$ from the centre of the instrument FOV. All observations were performed from approximately the end of February 2003 to the beginning of April 2006. ISGRI images for each pointing were generated in the 20-60 keV band using the ISDC offline scientific analysis software OSA version 5.1. Count rates at the position of the source were extracted from individual images to provide the source light curve from which a total of 19 flares were identified using the criterium described in Sect. 3. Then an ISGRI spectrum $(20-60 \mathrm{keV})$ of each flare was extracted and analysed; in one case (flare N. 18 in Table 1), the source was inside the JEM$\mathrm{X}$ FOV, so that low-energy spectral data could also be obtained over the 4-20 keV band. To further study the soft X-ray properties of the source, we used X-ray data collected with XRT (X-ray Telescope) on board the Swift satellite (Gehrels et al. 2004) whenever available. From the Swift archive, we found that IGR J16479-4514 was observed four times during the period August-October 2005. Table 2 reports the corresponding exposure for each observation, date, X-ray flux and luminosity (1-9 keV) as estimated using an absorbed power law fit to the XRT data. The XRT data reduction was performed according to the processes described in Landi et al. (2007). All spectral analysis reported in the paper was performed using Xspec version 11.3; uncertainties are given at the $90 \%$ confidence level for one single parameter of interest.

\section{Timing analysis}

The ISGRI long-term light curve $(20-60 \mathrm{keV})$ of IGR J16479-4514 on ScW timescale is shown in Fig. 1, where the black line represents the $2 \sigma$ upper limit at the $\mathrm{ScW}$ level $\left(\sim 10 \mathrm{mCrab}\right.$ or $\left.1.2 \times 10^{-10} \mathrm{erg} \mathrm{cm}^{-2} \mathrm{~s}^{-1}\right)$. Most of the time the source is not significantly detected at the ScW level, and it is below the instrumental sensitivity of ISGRI. It undergoes fast X-ray flares sporadically. In particular we considered those outbursts having a peak flux greater than $\sim 30 \mathrm{mCrab}$ or $3.6 \times 10^{-10} \mathrm{erg} \mathrm{cm}^{-2} \mathrm{~s}^{-1}(20-60 \mathrm{keV})$. This peak flux value is represented in Fig. 1 by the broken line and corresponds to a source significance detection equal to or greater than $\sim 6 \sigma$ in the single $\mathrm{ScW}$ containing the peak of the flare. By applying this criterium, a total of 19 fast X-ray flares have been detected and are listed in Table 1, together with the date of the peak emission, approximative duration of the entire flaring activity, flux, and luminosity at the peak (20-60 keV). In particular, ten new flares are reported here for the first time (N. 6, 7, 10, 11, 12, 14, 15, 16, 17, 19 in Table 1). We note that Walter \& Zurita (2007) report a total of 38 flares (27 short and 11 long) from this source although no detailed analysis of individual flares is presented. The difference in the number of flares is very likely due to a different total observing time and flare definition. In particular, we adopt a conservative peak flux 


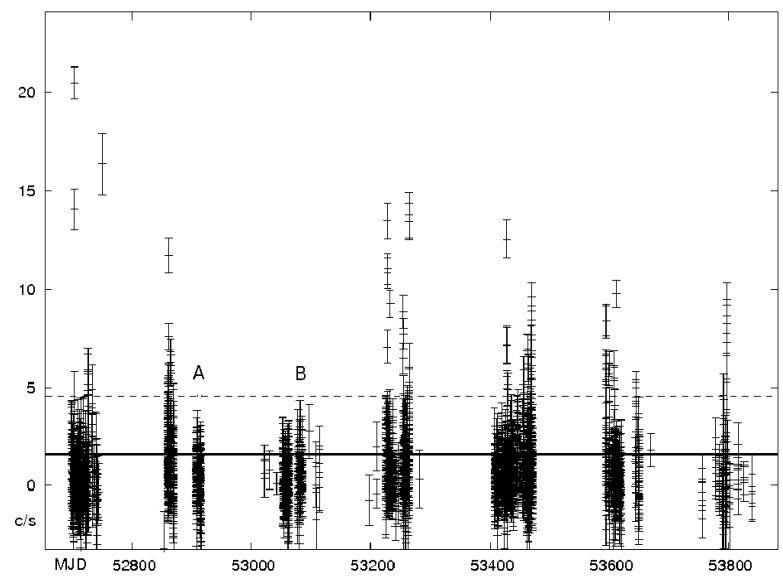

Fig. 1. ISGRI long term light curve (20-60 keV) of IGR J16479-4514. Time and flux axis are in MJD and count $\mathrm{s}^{-1}$, respectively. Each data point represents the average flux during one $\mathrm{ScW}(\sim 2000 \mathrm{~s})$.
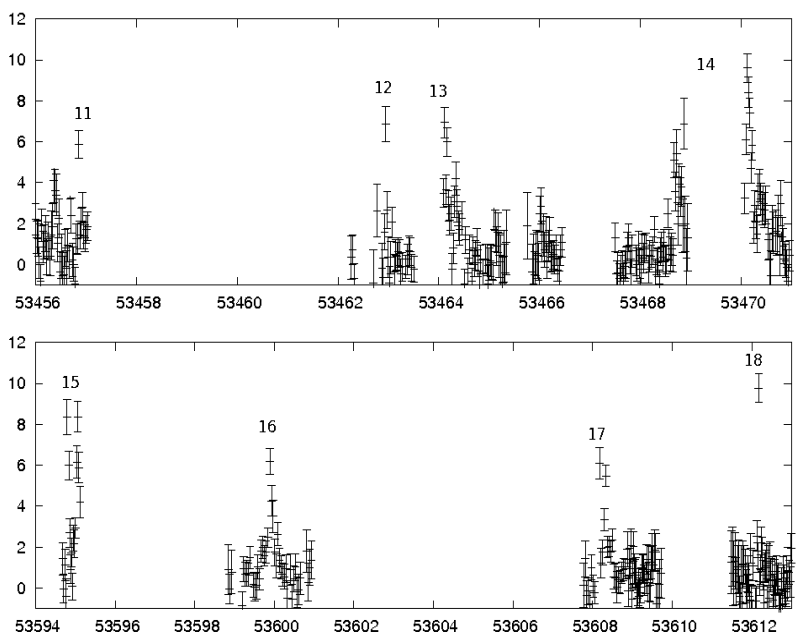

Fig. 2. Zoomed views of the light curves of flares from N. 11 to 18 . Time and flux axis are in MJD and count $\mathrm{s}^{-1}$, respectively. Each data point represents the average flux during one $\mathrm{ScW}(\sim 2000 \mathrm{~s})$.

threshold for flare recognition to pick up flare bright enough to extract a meaningful ISGRI spectrum.

On the basis of Table 1, we note that the source occasionally displays activity over a period of a few days, although the typical flare duration is only a few hours. Following the classification between short and long flares by Walter \& Zurita (2007), we report 12 flares of the first type and 7 of the second, i.e. a similar ratio to what they report. The typical peak flux is in a narrow range $\sim 40-80 \mathrm{mCrab}(20-60 \mathrm{keV})$ but occasionally much brighter flares occur. A detailed analysis of Fig. 1 indicates that the typical flare recurrence time is $\sim 1-2$ days: 19 flares were detected over a total exposure of $\sim 23$ days while more flares $(\sim 4)$ were occasionally seen over a $\sim 9$ days period (see examples in Fig. 2). This agrees quite well with the average recurrence time reported by Walter \& Zurita (2007). To search for real evidence of periodicity, we also used the Lomb-Scargle method with the fast implementation of Press \& Rybicki (1989) and Scargle (1982), but no indication of periodicity was found in the range 1-300 days. We also searched a $1 \mathrm{~s}$ bin time ISGRI light curve of all brightest outbursts in Table 1 (N. 1, 6, 9, 10, 18) for pulsations, but none were found.

A deeper inspection of Fig. 1 shows that the source occasionally enters long periods of very low flux level (for example, blocks A and B in Fig. 1), and we tentatively associate them

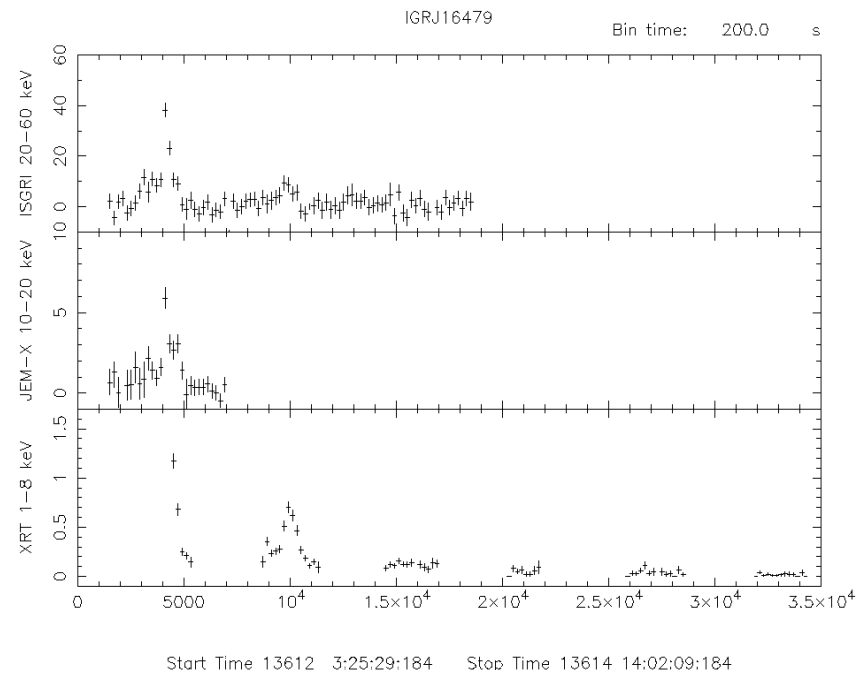

Fig. 3. ISGRI (top), JEM-X (middle) and Swift/XRT (bottom) simultaneous light curves of the flare N. 18 in Table 1 . The bin time is $200 \mathrm{~s}$.

to the source quiescence that must be below $\sim 10 \mathrm{mCrab}$ (20-60 keV).

Flare N. 18 in Table 1 is particularly interesting because it was discovered by Swift/BAT (15-50 keV) which promptly triggered the $S$ wift/XRT observation $(1-8 \mathrm{keV})$ that caught the flare only during its decay phase (Kennea et al. 2005). Here we report for the first time the simultaneous JEM-X and ISGRI detection of such flare, which provides a light curve (200 s bin time) in two different energy bands: $10-20 \mathrm{keV}(\mathrm{JEM}-\mathrm{X})$ and $20-60 \mathrm{keV}$ (ISGRI) as reported in Fig. 3.

During the four Swift/XRT observations (see Table 2), IGR J16479-4514 showed flaring activity only at the beginning of OBS1 (see bottom Fig. 3) where two X-ray flares are evident. Apart from the first flare previously cited, a second one started $\sim 4000$ s later with a duration of $\sim 1000$ s. It was outside the JEM-X FOV, while it may be present in the ISGRI light curve (see top Fig. 3) however without sufficient statistical significance for a secure claim because it was too faint (average flux $\sim 2 \times 10^{-11} \mathrm{erg} \mathrm{cm}^{-2} \mathrm{~s}^{-1}, 1-9 \mathrm{keV}$ ). No more flaring activity was detected in the remaining part of OBS1 or in the following Swift/XRT pointings (OBS2/3/4), and the source appeared to have reached its likely quiescent state with a typical $1-9 \mathrm{keV}$ luminosity of $\sim 10^{34} \mathrm{erg} \mathrm{s}^{-1}$ (see Table 2).

\section{X-ray spectral analysis}

\subsection{Flares}

To date ISGRI spectral information on flares from SFXTs is sparse; however, in the case of IGR J16479-4514 the large number of bright flares allows a proper study. For the majority of flares reported in Table 1 we were able to extract an ISGRI spectrum and perform a fit with two different spectral models: power law and bremsstrahlung. Spectral parameters, $\chi_{v}^{2}$ and the corresponding degree of freedom (d.o.f.) are all listed in Table 1. A distinction between these two models on a statistical basis is not possible because all fits give acceptable and comparable $\chi_{v}^{2}$; the bremsstrahlung temperatures and power law indices fall in a narrow range of $k T=21-46 \mathrm{keV}$ and $\Gamma=2.2-3$, and this suggests constancy in shape (but not flux) of the source spectra from flare to flare. Bearing this in mind and to improve the statistics, ISGRI spectra from all flares were fit together using the two models previously adopted. The bremsstrahlung model 
provided $k T=27_{-3.6}^{+3.2} \mathrm{keV}\left(\chi_{v}^{2}=0.9,109\right.$ d.o.f. $)$, while the power law gave $\Gamma=2.66 \pm 0.13\left(\chi_{v}^{2}=0.95,109\right.$ d.o.f. $)$.

Next we analysed in detail flare N. 18 using all avaliable data. An absorbed power law fit to the Swift/XRT data alone (relative to only the first flare at the bottom of Fig. 3) provided a flat photon index and absorption $N_{\mathrm{H}}$ (see Table 2) in excess of the galactic value, which is $2.1 \times 10^{22} \mathrm{~cm}^{-2}$ along the line of sight (Dickey \& Lockman 1990). JEM-X data alone are also well fit by a power law with photon index $\Gamma=2.2 \pm 0.2$, a value very similar to that found by ISGRI. A change in shape is clearly evident going from Swift/XRT to JEM-X/ISGRI, possibly due to extra absorption or to a high energy cutoff. We subsequently performed the broad band spectral analysis over the 2-60 keV energy range. A simple power law model poorly fit the data since the residuals clearly show the presence of absorption at soft X-rays. In fact, an absorbed power law provides a meaningful fit to the data $\left(\chi_{v}^{2}=0.9,154\right.$ d.o.f. $)$ with $\Gamma=2.5 \pm 0.2$ and $N_{\mathrm{H}}=16 \pm 3 \times 10^{22} \mathrm{~cm}^{-2}$ and Fig. 4 (top) displays this unfolded broad band spectrum. No cut-off is statistically required in the data fit. We introduced a constant to take possible miscalibrations into account between the instruments and also to compensate for the incomplete coverage of Swift/XRT. The XRT/JEM-X and JEM-X/ISGRI constants were found to be $0.13_{-0.02}^{+0.25}$ and $1_{-0.2}^{+0.3}$, respectively; the former is low because Swift/XRT only detected the flare during its decay phase, while JEM-X/ISGRI detected it throughout the entire duration of the event. The total $N_{\mathrm{H}}$ inferred from the absorbed power law broad band fit is much higher than that obtained from individual Swift/XRT spectral fits. Such high $N_{\mathrm{H}}$ could explain why the Swift/XRT spectrum have a rather hard photon index compared to the steeper JEM-X/ISGRI spectra. Moreover, an absorbed bremsstrahlung model fits the data equally well $\left(\chi_{v}^{2}=1.05,154\right.$ d.o.f., $\left.k T=19_{-4.3}^{+6.3}, N_{\mathrm{H}}=9_{-2}^{+2.5} \times 10^{22} \mathrm{~cm}^{-2}\right)$, and the values of the two constants are very similar to those previously found.

\subsection{Quiescence}

From the long-term light curve of IGR J16479-4514, we individuated a total of $\sim 530$ pointings during which the source is not significantly detected in any individual $\mathrm{ScW}$ (see blocks A and B in Fig. 1); however, a mosaic of all these ScWs provided a clear detection of the source at $\sim 9 \sigma$ level in the $20-60 \mathrm{keV}$ band. The fluxes for spectral analysis were extracted from the location of IGR J16479-4514 in fine band mosaics of all $530 \mathrm{ScWs}$ and a spectrum was produced according to the processes described in Bird et al. (2007). This spectrum is equally well fit using a power law $\left(\Gamma=2.5 \pm 1, \chi_{v}^{2}=0.2,4\right.$ d.o.f. $)$ and a bremsstrahlung $\left(k T=30_{-14}^{+47} \mathrm{keV}, \chi_{v}^{2}=0.2\right.$, 4 d.o.f.). The average $20-60 \mathrm{keV}$ flux and luminosity are $\sim 1.7 \times 10^{-11} \mathrm{erg} \mathrm{cm}^{-2} \mathrm{~s}^{-1}$ and $\sim 5 \times 10^{34} \mathrm{erg} \mathrm{s}^{-1}$. Walter \& Zurita (2007) report a similar ISGRI quiescent flux of $\sim 1.54 \times 10^{-11} \mathrm{erg} \mathrm{cm}^{-2} \mathrm{~s}^{-1}$, confirming that such a value is the lowest hard X-ray emission level detected from the source to date.

The soft X-ray properties of the quiescence as detected by Swift/XRT can be safely associated to OBS2/3/4 and to the final part of OBS1. All spectra pertaining to these observations are best fit by an absorbed power law model (see Table 2), and the values of the photon index are compatible within the uncertainties, indicating that the source may be characteriased by the same rather hard spectral shape during quiescence and also during the decay phase of flare N. 18. We checked for variability in the spectral indices between OBS4 and OBS1/quiescence by

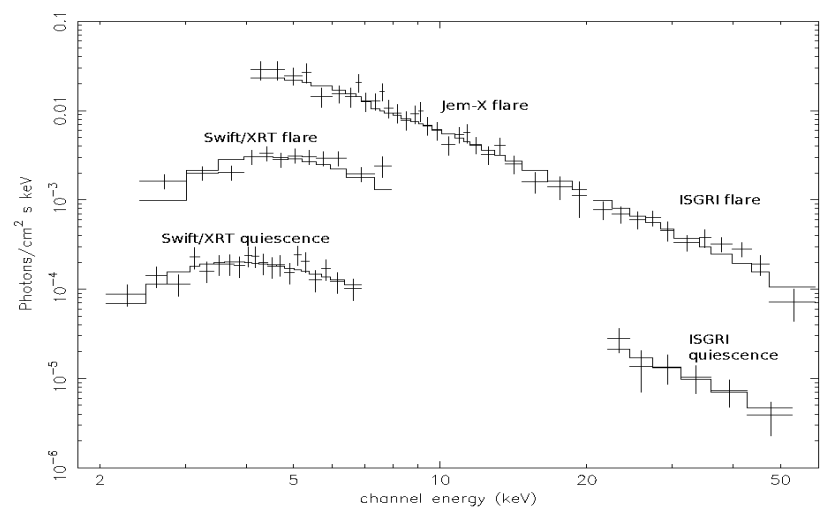

Fig. 4. Unfolded broad band spectrum $(2-60 \mathrm{KeV})$ of flare N.18 in Table 1 (top) and of the quiescent emissiom (bottom).

fixing the $N_{\mathrm{H}}$ value of the first observation to that of the second; by doing so, no variability has been found since the photon index assumes an almost identical value of $\Gamma=0.9 \pm 0.3$.

We combined the Swift/XRT spectrum of OBS4 with that of ISGRI in quiescence to obtain broad band energy information over the $2-60 \mathrm{keV}$ band. The underlyng assumption is that the spectral shape of the source did not change during the time interval between the Swift/XRT and the IBIS observations, which is reasonable given the constancy in shape seen both by IBIS and Swift/XRT. The best fit is provided by an absorbed power law model $\left(\chi_{v}^{2}=0.5,22\right.$ d.o.f. $)$ with $\Gamma=2.2 \pm 0.75$ and a total $N_{\mathrm{H}}=9_{-3.5}^{+4.0} \times 10^{22} \mathrm{~cm}^{-2}$. The cross calibration constant between the two instruments is $0.5_{-0.3}^{+1}$. Figure 4 (bottom) displays the unfolded broad band spectrum. Also in this case, the bremsstrahlung provided a comparably good fit $\left(\chi_{v}^{2}=0.4\right.$, 22 d.o.f.); however, the temperature was not well constrained $(k T \sim 20 \mathrm{keV})$. We point out that the broad band X-ray spectral shapes of the source in quiescence and during flaring activity are very similar, as can be clearly noted in Fig. 4.

\section{Discussion and conclusions}

In this paper, we present results from a long-term monitoring of IGR J16479-4514 with detailed information on 19 bright flares, 10 of which are newly discovered. The flares were detected typically every $\sim 1-2$ days, this makes IGR J16479-4514 the SFXT with the highest duty cycle seen so far. However, the source is occasionally in a quiescent state, and the longest period of inactivity sampled by our monitoring is $\sim 12$ days.

The typical flare duration is only a few hours but occasionally longer flaring activity has been detected. Our detailed X-ray spectral analysis shows that the shape of the source in quiescence and during flares is identical (i.e. a rather steep power law with $\Gamma \sim 2.6$ ), despite large excursions in flux. Moreover, the source is always detected when observed by an X-ray instrument having sufficient sensitivity, such as Swift/XRT, with a 1-9 keV flux (luminosity) of $\sim 10^{-12} \mathrm{erg} \mathrm{cm}^{-2} \mathrm{~s}^{-1}\left(\sim 10^{34} \mathrm{erg} \mathrm{s}^{-1}\right)$. Since the typical peak flux of flares is $\sim 10^{-9} \mathrm{erg} \mathrm{cm}^{-2} \mathrm{~s}^{-1}$, IGR J16479-4514 must accrete over a dynamic range of $\sim 3500$. More important, its quiescence is higher than what is typical of other SFXTs by about two orders of magnitude (Negueruela et al. 2006), which raises the possibility that, during the quiescence of IGR J16479-4514, the compact object is still close to the supergiant donor star, so it is still accreting a significant amount of material from its wind but not in the form of clumps as during fast $\mathrm{X}$-ray flares. This would explain how the same spectral shape is seen both in quiescence and during flares, since the emission 
mechanism should be the same, i.e. accretion onto the compact object. Consecutively, the system should be characterised by a small orbital radius and weak eccentricity; otherwise, it would be in quiescence for longer intervals (>12 days) and with lower $\mathrm{X}$-ray luminosity than those actually observed.

Considering that the quiescent X-ray luminosity of IGR J16479-4514 is intermediate between that of other SFXTs and classical persistent SGXBs, we suggest that this system is a transition object between the two classes. This supports the idea that there is a continuum of behaviour between SFXTs and classical SGXBs (Negueruela et al. 2008b). What differentiates such systems is most likely the different wind properties and/or orbital parameters, i.e. orbital radius, orbital period, and eccentricity (Negueruela et al. 2008a; Chaty et al. 2008). It is important to point out that in the literature there are at least three other unidentified X-ray sources that could be similar to IGR J16479-4514, i.e. IGR J16195-4945 (Sguera et al. 2006; Walter \& Zurita 2007; Tomsick et al. 2006), IGR J16418-4532 (Sguera et al. 2006; Walter et al. 2006), and XTE J1743-363 (Sguera et al. 2006; Walter \& Zurita 2007).

The accumulation of exposure time and the longer temporal coverage of the source by IBIS is very likely to increase the possibility of discovering the orbital period of IGR J16479-4514, which could provide key information for studying and understanding the physical reasons behind its very unusual X-ray behaviour.

Acknowledgements. The authors acknowledge the ASI financial support via grant ASI-INAF I/088/06/0, ASI-IANF I/023/05/0.

\section{References}

Bird, A. J., Malizia, A., Bazzano, A., et al. 2007, ApJS, 170, 175 Chaty, S., Rahoui, F., Foellmi, C., et al. 2008, A\&A, 484, 783 Dickey, J. M., \& Lockman, F. J. 1990, ARA\&A, 28, 215 Gehrels, N., Chincarini, G., Giommi, P., et al. 2004, ApJ, 611, 1005 Kennea, J. A., Pagani, C., Markwardt C., et al. 2005, ATEL, 599 Landi, R., Masetti, N., Morelli, L., et al. 2007, ApJ, 669, 109 Laurent, P., Paul, J., Denis, M., et al. 1995, A\&A, 300, 399 Lebrun, F., Leray, J. P., Lavocat, P., et al. 2003, A\&A, 411, 141 Leyder, J. C., Walter, R., Lazos, M., et al. 2007, A\&A, 465, 35 Lund, N., Jorgentsen, C., Westergaard, N. J., et al. 2003, A\&A, 411, L231

Markwardt, C. B., Swank, J. H., \& Marshall, F. E. 1999, IAUC, 7120

Molkov, S., Mowlavi, N., Goldwurm, A., et al. 2003, ATEL, 176

Negueruela, I., Smith, D., Reig, P., et al. 2006, ESA SP-604, 165

Negueruela, I., Torrejon, J., Reig, P., et al. 2008a, A population explosion: the nature and evolution of X-ray binaries in diverse environments, AIP Conf. Proc., 1010, 252

Negueruela, I., Smith, D., Torrejon, J., et al. 2008b, 6th Integral Workshop The obscured Universe, ESA SP-622, 255

Press, W. H., \& Rybicki, G. B. 1989, ApJ, 338, 277

Scargle, J. D. 1982, ApJ, 263, 835

Rahoui, F., Chaty, S., Lagage, P., et al. 2008, A\&A, 484, 801

Sguera, V., Barlow, E. J., Bird, A. J., et al. 2005, A\&A, 444, 221

Sguera, V., Bazzano, A., Bird, A. J., et al. 2006, ApJ, 646, 452

Staubert, R. K., reykenbohm, I., et al. 2004, ESA SP-552, 259

Tomsick, J. A., Chaty, S., Rodriguez, J., et al. 2007, ApJ, 647, 1309

Ubertini, P., Lebrun, F., Di Cocco, G., et al. 2003, A\&A, 411, L131

Walter, R., \& Zurita Heras, J. 2007, A\&A, 476, 335

Walter, R., Zurita Heras, J., Bassani, L., et al. 2006, A\&A, 453, 133

Winkler, C., Courvoisier, T., Di Cocco, G., et al. 2003, A\&A, 411, L1 